Cahiers $d u$ MONDE RUSSE

\section{Cahiers du monde russe}

Russie - Empire russe - Union soviétique et États indépendants

$47 / 4 \mid 2006$

Varia

\title{
Natal'ja Viktorovna Suržikova, Inostrannye voennoplennye Vtoroj mirovoj vojny na Srednem Urale
}

\section{Vanessa Voisin}

\section{(2) OpenEdition}

1 Journals

\section{Édition électronique}

URL : https://journals.openedition.org/monderusse/6751

DOI : 10.4000/monderusse.6751

ISSN : $1777-5388$

Éditeur

Éditions de l'EHESS

\section{Édition imprimée}

Date de publication : 30 décembre 2006

Pagination : 865-869

ISBN : 978-2-7132-2098-2

ISSN : $1252-6576$

\section{Référence électronique}

Vanessa Voisin, « Natal'ja Viktorovna Suržikova, Inostrannye voennoplennye Vtoroj mirovoj vojny na Srednem Urale », Cahiers du monde russe [En ligne], 47/4 | 2006, mis en ligne le 03 juillet 2009, consulté le 03 septembre 2022. URL : http://journals.openedition.org/monderusse/6751 ; DOI : https://doi.org/10.4000/monderusse.6751

Ce document a été généré automatiquement le 3 septembre 2022.

Tous droits réservés 


\title{
Natal'ja Viktorovna Suržikova, Inostrannye voennoplennye Vtoroj mirovoj vojny na Srednem Urale
}

\author{
Vanessa Voisin
}

\section{RÉFÉRENCE}

Natal'ja Viktorovna SURŽIKOVA, Inostrannye voennoplennye Vtoroj mirovoj vojny na Srednem Urale, 1942-1956 gg. [Les prisonniers de guerre étrangers de la Seconde Guerre mondiale dans les camps du Moyen Oural, 1942-1956] Ekaterinbourg : Gumanitarnyj universitet, 2006, 500 p., 47 ill.

$1 \quad$ Natal'ja Suržikova, auteur de nombreuses contributions sur les prisonniers de guerre étrangers en URSS, offre ici une étude très sérieuse sur la détention, le travail des détenus étrangers incarcérés lors de la Seconde Guerre mondiale dans les camps du Moyen Oural, ainsi que sur les poursuites judiciaires dont ils ont été l'objet. L'auteur annonce un traitement transdisciplinaire du sujet, privilégiant les phénomènes de transfert et d'adaptation culturelle ainsi qu'un processus d'auto-identification de la société nationale. Cependant, l'ouvrage livre en fait une interprétation politique du sort réservé aux prisonniers de guerre étrangers en URSS. L'axe majeur est celui de la parenté avec le destin des Soviétiques du Goulag, en dépit d'évidentes différences liées au statut de ressortissants étrangers (plus que de prisonniers de guerre) de ces captifs. Cette approche explique les bornes chronologiques retenues ainsi que la structure, assez déséquilibrée, de l'ensemble. N. Suržikova récuse ainsi les catégories forgées par le pouvoir de l'époque, qui datait de 1950 la fin du rapatriement des prisonniers de guerre étrangers dans leur pays. La première partie de l'ouvrage est consacrée à la période où l'écrasante majorité de ces détenus n'est encore considérée que comme « prisonniers de guerre » et « internés » (1942-1949). Mais lui succède une seconde section sur les années 1949-1956, au cours desquelles leur statut se modifie: ceux qui restent sont des " condamnés », essentiellement pour crimes de guerre. Selon l'auteur, ce changement 
s'inscrit dans la continuité de l'attitude des autorités soviétiques à l'égard de cette population carcérale : non-respect des conventions internationales lié à une conception de la primauté du droit national sur l'international, mais en même temps traitement relativement privilégié de ces " otages " par rapport aux détenus nationaux du Goulag. $\mathrm{Si}$ les Soviétiques répriment et détiennent les ressortissants étrangers selon des modalités relevant d'une tradition établie depuis les années 1930, ils ne perdent jamais de vue la spécificité de ce contingent (considérations idéologiques, politiques et diplomatiques). Une dernière partie, beaucoup plus brève, sur l'utilisation de ces prisonniers dans l'économie régionale parachève cette démonstration. À l'instar des condamnés soviétiques pour crime politique, les détenus étrangers furent affectés aux industries déficitaires en main-d'œuvre et donc à un travail particulièrement pénible.

2 Procédons maintenant à une présentation détaillée de chacune des parties. L'étude s'ouvre sur un examen des conditions de détention des prisonniers étrangers dans les camps de l'Oural de 1942 à 1950. Comparées à celles des détenus soviétiques du Goulag, et compte tenu du contexte de guerre totale, celles-ci constituent « un acte d'humanisme inattendu de la part d'un pouvoir totalitaire », bien que les réglementations internationales relatives aux prisonniers de guerre n'aient pas été respectées. L'auteur fait ici surtout référence à l'obligation de leur assurer des conditions matérielles égales à celles de l'armée nationale, mais relève aussi d'autres infractions, telles que des condamnations sévères pour évasion sans circonstances aggravantes, ou encore l'ajournement de leur rapatriement comme mesure punitive.

3 La détention des prisonniers de guerre en URSS s'appuya de fait largement sur l'expérience accumulée depuis l'avènement du régime, plus spécialement depuis les années 1930. L'administration des camps en général, leur fonctionnement interne, les conditions de vie, la pratique de recrutement d'informateurs parmi les captifs, l'encouragement par les autorités d'organisations souterraines antifascistes.... sont autant d'éléments communs au Goulag soviétique et aux lieux de détention pour prisonniers de guerre.

4 Cependant, ces similitudes s'expliqueraient moins par une volonté politique d'assimilation ou d'identification que par la force d'inertie des pratiques d'isolement, d'exploitation et de "rééducation» d'une population carcérale de masse. On relève même, à propos du cantonnement de cette population dans l'Oural, un désaccord entre autorités centrales et régionales: la Direction régionale du commissariat du peuple à l'Intérieur appréhendait la concentration de ce type de détenus dans un espace industriel appelé à devenir de plus en plus stratégique avec l'évacuation des usines d'armement de l'ouest du pays. On peut d'ailleurs regretter que l'auteur n'approfondisse pas ce constat et qu'elle ne suive pas l'évolution de cette divergence de vues tout au long de la période étudiée. Car si cette opposition révèle certes un heurt entre considérations d'ordre économique (utiliser cette main-d'œuvre) d'une part, et d'ordre sécuritaire de l'autre, rien n'interdit de supposer des divergences de représentations plus essentielles entre centre et régions, autorités politiques et administrations policières.

5 C'est ici la question de la spécificité de ce contingent carcéral qui est en cause. $\mathrm{N}$. Suržikova en décline les caractéristiques sociologiques (âge moyen, nationalité, aptitude au travail, etc.), sans s'attarder sur les aspects culturels et anthropologiques pourtant si pertinents dans le cadre d'une telle étude. Elle évoque ainsi le débat sur l'origine des associations antifascistes dans les camps, et préfère à raison tirer une conclusion nuancée : des autorités très impliquées - voire instigatrices - dans la formation de ces 
groupes, et des détenus aux motivations partagées entre une conviction idéologique sincère et l'espoir d'un retour plus rapide dans leur pays. Mais elle ne s'intéresse pas aux «stratégies de survie » développées par des individus en milieu profondément étranger. L'analyse des relations entre détenus, ou entre captifs et geôliers, est à peine effleurée, alors même que le riche corpus de sources consultées laissait espérer des avancées dans ce domaine. De même, le déracinement vécu par des hommes détenus à des milliers de kilomètres de chez eux, dans un pays dont la langue leur est le plus souvent étrangère et l'idéologie a priori hostile, ne fait pas l'objet d'un traitement spécifique. L'auteur décrit différentes formes de contact entre civils soviétiques et détenus étrangers, à partir de documents très intéressants (témoignages, dossiers judiciaires, rapports administratifs), mais là encore sans approfondir cet aspect.

6 C'est en effet surtout la question de la qualité des conditions de détention, toujours par comparaison avec celles des détenus soviétiques du Goulag, qui guide sa lecture. Le cas du Moyen Oural lui permet de distinguer une amélioration de ces conditions au fur et à mesure que la situation militaire bascule en faveur des Alliés et qu'émerge la perspective d'une exploitation idéologique de ces futurs rapatriés (ou, a contrario, futurs condamnés pour crimes de guerre). L'efficacité de la mise en place, ex nihilo et en moins de trois ans, de camps "vivables» fonde en grande partie cette conclusion. Une étude précise des rations alimentaires démontre, à côté des aléas conjoncturels dont souffrirent aussi bien les civils soviétiques de l'arrière, des difficultés durables jusqu'en 1948, mais aussi un statut relativement privilégié du contingent étranger par rapport au contingent national, en dépit de la rapacité du personnel administratif. L'État soviétique s'employa réellement à garantir un "minimum» alimentaire, vestimentaire et médical à ces détenus, certes moins pour des raisons humanitaires que pour des raisons politiques et économiques.

7 La deuxième partie de l'ouvrage est consacrée à la période suivante (1950-1955), au cours de laquelle les prisonniers de guerre sont désormais détenus en tant que criminels (de droit commun, mais surtout de guerre). Ce point est extrêmement complexe, car aux aspects proprement juridiques se mêlent les facteurs politiques et diplomatiques. Évitant un traitement thématique, l'auteur choisit d'étudier simultanément ces différents aspects à travers l'exemple du plus important des camps pour prisonniers de guerre et internés condamnés d'URSS, le camp n476 du ministère de l'Intérieur (ch. 1, $2^{\mathrm{e}}$ partie). Des données très précises permettent de mettre en évidence les caractéristiques de cette population carcérale ainsi que de la politique répressive de l'Union soviétique. Cette politique, tout en conservant ses traits propres (acteurs et réactivité de ces acteurs aux impulsions du centre, chefs d'accusation: condamnations pour crimes contrerévolutionnaires commis par certains étrangers après leur capture, par exemple), ne se comprend en réalité que dans le contexte du début de la guerre froide. Les prisonniers de guerre deviennent un instrument aux mains du pouvoir soviétique. Ainsi celui-ci marque son désaccord avec la politique menée à l'ouest de l'Europe d'une part, et garde une masse d' "otages » européens dans un climat de refroidissement des relations entre anciens alliés de l'autre. N. Suržikova relève une accélération et une simplification des procédures à partir de 1947-1948, impulsées d'en haut, alors même que l'URSS avait devancé le droit international en matière de poursuites contre les criminels de guerre (le décret du Présidium du Soviet Suprême de l'URSS relatif aux crimes de guerre datait du 19 avril 1943). Ce point lui-même pose problème, car la formulation de ce décret contre "les malfaiteurs fascistes et leurs acolytes" trahissait une lecture idéologique de la répression des crimes de guerre et surtout péchait par imprécision au sujet du corps du 
crime, laissant libre cours aux interprétations extensives. Des exemples concrets de dossiers d'instruction démontrent l'exploitation de ce flou juridique ainsi que, par ailleurs, une nette dérive répressive (condamnations sans preuves, rejet systématique des sentences, etc.). Quatre condamnés étrangers sur cinq auraient ainsi été recevables pour une procédure de réhabilitation en tant que victimes de répressions politiques, au même titre que les victimes soviétiques du régime stalinien des années 1930-1940.

8 Le destin des condamnés pour crimes de guerre en URSS a donc bien plus en commun avec celui des zek du Goulag stalinien qu'avec celui des prisonniers de guerre soviétiques en Allemagne pendant la guerre. Cette thèse trouve confirmation dans l'étude de leurs conditions de détention et de rapatriement (ch. 2). L'incarcération de cette catégorie de condamnés dans les camps spéciaux du ministère de l'Intérieur impliquait un traitement en tant «qu'éléments socialement dangereux » particulièrement sévère, mais assimilable à celui des détenus soviétiques pour crime politique. À ceci près que la nationalité des détenus leur assurait un certain nombre de privilèges par rapport à leurs homologues soviétiques (droit de plainte au sujet de la détention, droit de correspondance avec leurs proches...). Enfin, le facteur diplomatique, qui tendait à jouer contre eux sous Staline, devint un avantage après sa mort. Il fallut certes deux ans pour que la direction soviétique décide de rendre presque tous les ex-prisonniers de guerre à leurs pays d'origine (été 1955), mais du moins ceux-ci ne purgèrent-ils pas la totalité de leur peine (souvent entre 15 et 25 ans), au contraire des Soviétiques.

9 La troisième et dernière partie traite de l'exploitation économique des prisonniers de guerre étrangers, un aspect central de la politique soviétique à leur égard. L'un des faits saillants que relève l'auteur est la primauté du principe de nécessité sur le principe institutionnel et administratif. Ainsi, l'emploi de ces détenus dans les entreprises régionales déficitaires en main-d'œuvre plutôt que sur les sites du NKVD/MVD s'oppose radicalement aux pratiques en usage pour ce qui concerne les Soviétiques du Goulag. L'auteur s'intéresse ensuite aux conditions de travail, de garde, de respect des règles de sécurité et à la productivité de ce contingent. Ces paramètres mettent en évidence un souci d'utilisation rationnelle des ressources humaines qui prit même le pas dès 1947 sur l'objectif de "rééducation" idéologique, mais se heurta aux contradictions de la politique soviétique à leur égard. Ainsi, le passage du statut de prisonnier de guerre à celui de condamné, en ajournant les perspectives de rapatriement, porta grand tort à la stimulation des détenus malgré l'existence d'un système de rémunération en fonction de la norme de travail effectuée. Pour autant, les conclusions de N. Suržikova infirment la représentation dominante de l'utilisation de cette main-d'œuvre dans l'Oural, région très représentative de la situation globale des prisonniers de guerre en URSS. Ceux-ci furent massivement employés dans l'économie nationale dès 1942 et si leur productivité peut être mise en doute pendant les années de ravitaillement difficile, elle s'améliore nettement avec la conjoncture économique globale.

10 Ainsi, malgré de nombreuses données très concrètes sur l'organisation des lieux de détention, le profil sociologique des prisonniers, leurs conditions de vie, malgré le recours à un riche corpus de sources très diversifiées permettant une approche microhistorique, voire anthropologique et culturelle, l'auteur ne cherche pas ici à proposer une histoire sociale des détenus étrangers de l'Oural, et encore moins une histoire culturelle ou de la vie quotidienne. On peut parfois le regretter, étant donné la qualité des sources consultées et l'intérêt du sujet. Néanmoins, N. Suržikova le souligne ellemême: un grand nombre des aspects traités mériterait à eux seuls des ouvrages 
spécifiques excédant les limites du présent propos. Mais on comprend mal alors le choix des annexes, abondantes, qui portent davantage sur les points sciemment laissés de côté par l'ouvrage que sur sa problématique de fond : un dossier photographique sur le camp $n^{\circ} 476$ livre un aperçu vivant de l'existence carcérale, tandis que les témoignages de détenus jugés les plus intéressants sont reproduits sur une soixantaine de pages. A contrario, on déplore l'absence de simples cartes de la région à l'époque traitée, toujours bienvenues dans une monographie régionale, ainsi que du texte des actes juridiques et politiques les plus importants. Ces petits bémols n'ôtent rien à un travail tout à fait remarquable par le volume de ses sources, la clarté générale du propos et l'honnêteté intellectuelle de l'analyse. 